\title{
Article \\ DNA Dosimetry with Gold Nanoparticle Irradiated by Proton Beams: A Monte Carlo Study on Dose Enhancement
}

\author{
Ngoc Han Huynh ${ }^{1}$ and James C. L. Chow ${ }^{2,3, *(1)}$ \\ 1 Department of Physics, Ryerson University, Toronto, ON M5B 2K3, Canada; ngochan.huynh@ryerson.ca \\ 2 Radiation Medicine Program, Princess Margaret Cancer Centre, University Health Network, \\ Toronto, ON M5G 1X6, Canada \\ 3 Department of Radiation Oncology, University of Toronto, Toronto, ON M5T 1P5, Canada \\ * Correspondence: james.chow@rmp.uhn.ca; Tel.: +1-416-946-4501
}

Citation: Huynh, N.H.; Chow, J.C.L. DNA Dosimetry with Gold

Nanoparticle Irradiated by Proton

Beams: A Monte Carlo Study on Dose Enhancement. Appl. Sci. 2021, 11, 10856. https://doi.org/10.3390/ app112210856

Academic Editors: Lionel MAURIZI and Qi-Huang Zheng

Received: 3 October 2021

Accepted: 16 November 2021

Published: 17 November 2021

Publisher's Note: MDPI stays neutral with regard to jurisdictional claims in published maps and institutional affiliations.

Copyright: $\odot 2021$ by the authors. Licensee MDPI, Basel, Switzerland. This article is an open access article distributed under the terms and conditions of the Creative Commons Attribution (CC BY) license (https:// creativecommons.org/licenses/by/ $4.0 /)$.

\begin{abstract}
Heavy atom nanoparticles, such as gold nanoparticles, are proven effective radiosensitizers in radiotherapy to enhance the dose delivery for cancer treatment. This study investigated the effectiveness of cancer cell killing, involving gold nanoparticle in proton radiation, by changing the nanoparticle size, proton beam energy, and distance between the nanoparticle and DNA. Monte Carlo (MC) simulation (Geant4-DNA code) was used to determine the dose enhancement in terms of dose enhancement ratio (DER), when a gold nanoparticle is present with the DNA. With varying nanoparticle size (radius $=15-50 \mathrm{~nm}$ ), distance between the gold nanoparticle and DNA $(30-130 \mathrm{~nm})$, as well as proton beam energy $(0.5-25 \mathrm{MeV})$ based on the simulation model, our results showed that the DER value increases with a decrease of distance between the gold nanoparticle and DNA and a decrease of proton beam energy. The maximum DER (1.83) is achieved with a $25 \mathrm{~nm}$-radius gold nanoparticle, irradiated by a $0.5 \mathrm{MeV}$ proton beam and $30 \mathrm{~nm}$ away from the DNA.
\end{abstract}

Keywords: radiosensitizer; DNA dosimetry; gold nanoparticles; cancer therapy; dose enhancement; Monte Carlo simulation; nanoparticle-enhanced radiotherapy

\section{Introduction}

Worldwide, millions of people are diagnosed with cancer each year, and roughly every sixth death in the world is due to cancer [1]. In fact, cancer is responsible for $30 \%$ of all death in Canada, making it the number one leading cause of death in the nation [1]. According to the Public Health Agency of Canada, in 2015, about 2.1 million Canadians aged 12 years and older have been diagnosed with cancer at some point during their lifetime. This statistic has increased to $20 \%$, approximately 1 in 5 Canadians, to be diagnosed with cancer by 70 years of age, making cancer the leading cause of death in Canada [1]. As the nation's population increases and the average life expectancy improves with the rise of new technologies, the number of cancer cases and deaths are becoming more prominent and expected to continue to grow over the coming decades [2].

Radiotherapy is one of the most common types of cancer treatment. The primary purpose of radiotherapy is to kill cancer cells by irradiating a specific dose of ionizing radiation onto the target tumour [3]. The radiation can either damage the cancer cells directly or indirectly at the deoxyribonucleic acid (DNA) level. The most apparent consequence of the practice is unwanted damage to the surrounding healthy cells, causing side effects that are intensely unpleasant for the patient. Therefore, during treatment planning, limited radiation doses are given to minimize the undesirable radiation effects. Such limitation lowers the overall effectiveness of radiotherapy. Recently, with recent advances of heavy atom radiosensitizers as an effective dose enhancer for the tumour and contrast agent in medical imaging, a maximum radiation dose can be delivered to the tumour while keeping the dose at the surrounding normal tissue to a minimum [4-6]. Heavy atom radiosensitizers are adjunctive agents that make cancer cells more susceptible to radiation, 
designed to improve cancer cell killing while having much less of an effect on the surrounding healthy tissue [7]. Nanoparticles are defined as particles with a radius dimension of about between 1 and $100 \mathrm{~nm}$. In many studies, gold nanoparticles have proven to be an excellent material for radiosensitizing. This is due to the high atomic number $(Z=79)$ of a gold nanoparticle, which can absorb more low-energy radiation (e.g., $\mathrm{kV}$ photon) than soft tissue, its biocompatibility, and it being easy to manufacture and be delivered to the patient $[8,9]$. Since tumour requires large amounts of blood vessels to proliferate rapidly, cancer cells stimulate angiogenesis. As the tumour vasculature is leaky, it enables nanoparticles injected in the bloodstream to have an easier time entering the tumour tissues. This process can be explained by using the enhanced permeability and retention effect, which is the property by which molecules of certain sizes (usually liposomes, nanoparticles and macromolecular drugs) accumulate in tumour tissue much more than they do with normal tissues [10]. Once the nanoparticles are delivered into the tumour volume, they absorb high-energy radiation and emit secondary electrons through interactions between radiation and the cancer cell medium [11]. These additional electrons then damage the tumour DNA alongside ionizing radiation, adding more dose deposition at the tumour cells. To date, gold nanoparticle-enhanced radiotherapy has become a new cancer treatment modality with many dosimetry, preclinical and clinical studies published [12-17].

Radiotherapy uses high-energy ionizing radiation or particles to damage cancer cells at the DNA scale to stop them from growing and dividing [18]. Depending on its ability to ionize matter such as the DNA strand, ionizing particles can be divided into two categories, namely, directly ionizing and indirectly ionizing particles. For directly ionizing particles, they deposit their energy in the tissue through direct Coulomb interactions between the charged particle and orbital electrons of atoms in the DNA molecules. Directly ionizing particles can "directly" damage the target cancer cells without relying on the production of secondary electrons. On the other hand, indirectly ionizing radiations, such as photons and neutrons, deposit their energy in the cancer cells through a two-step process. First, the uncharged particle travels through tissue and creates charged particles (e.g., photons release electrons or positrons, and neutrons release protons or heavier ions) from the molecules they interact with within the cell medium. Then, the charged particles move to the DNA to produce damage [19].

Nowadays, the use of megavoltage photon is the most commonly used method when treating cancer tumours with external beam radiation. A photon beam can be produced from radioactive sources such as Cobalt-60 or a treatment delivery system such as a linear accelerator. However, the most notable downside to photon therapy is the unwanted damage to the healthy tissues or critical organs in the patient. Usually, the target tumour is located at a certain depth inside the patient. When a photon beam is being irradiated, it deposits most of its energy at the entrance point (surface of the skin) and continuously loses its energy as the beam travels through the patient towards the tumour. The photon beam finally exits through the other side of the patient. In summary, healthy tissues located in front and behind the tumour inevitably receive some radiation dose, resulting in a certain amount of healthy cell death. In charged particle therapy, such as with protons, the beam characteristic of Bragg peak, where proton beam deposits most of its energy at the end of its path, gives rise to three crucial advantages compared to photon therapy. These advantages are the low entrance dose when the proton beam comes in contact with the patient, a large deposited dose at the tumour location and almost no exit dose [20]. Therefore, there is less damage to the healthy tissue of the patient, though both photon and proton therapy still have unnecessary doses at the healthy cells located along the beam path to the tumour.

In this study, we investigated the DNA dosimetry of the cancer cell in the presence of a gold nanoparticle irradiated by proton beams. Monte Carlo (MC) simulation was used to predict the dose enhancement by the addition of a gold nanoparticle in the proton irradiation [21-25]. The enhancement of DNA damage can be estimated by the dose enhancement ratio (DER), which is defined as the ratio of the dose at the DNA with addition of a gold nanoparticle to the dose at the DNA without the gold nanoparticle [26]. 
By examining the MC results, we can investigate the dependences of DER on different irradiation variables, namely, the nanoparticle size, distance between the nanoparticle and DNA and proton beam energy.

\section{Materials and Methods}

\subsection{Monte Carlo Simulation}

MC simulation is a mathematical method based on repeated random samplings to obtain numerical results, and it is useful to solve some physical and mathematical problems when it is difficult to use other approaches. In medical biophysics, MC simulation is often used to predict radiation dosimetry [27]. Since it is difficult to visualize how radiation behaves when particles radiate to the human body without computational technology, having a simulation before the actual therapy can help determine the dose distribution of the patient in radiotherapy. The MC simulation is believed to be the benchmark of predicting dose in radiotherapy [28].

In this study, MC simulation was used to predict the dosimetry of DNA with a gold nanoparticle irradiated by proton beams. The Geant4-DNA code was used in this nanodosimetric simulation. Geant4 (Geometry and Tracking) is a toolkit for the simulation of the passage of particles through matter, developed by CERN [29]. Geant4-DNA, an extension of the program to model biological damage induced by ionizing particles at the DNA scale using MC simulation, is written in C++ code working on the CMake platform [29,30]. The toolkit has the capability of making the geometry of the system, materials involved, tracking of particles through materials, the response of detector components, visual of the detector and particle trajectories, and other relevant aspects. The program is from open source and can be accessed through the Geant4 Virtual Machine provided by the Center d'Etudes Nucléaires de Bordeaux-Gradignan [31]. Geant4 uses CMake3.3 or higher to run the code, $\mathrm{C}++$ compiler and standard library supporting $\mathrm{C}++11$ standard for programming. Geant4-DNA provides a virtual machine containing CentOS Linux, the latest version of Geant4 (version 10.7 was installed for this study), visualization tools, analysis tools, and other utilities are packaged with the Virtual Machine (VMware Workstation) [32].

\subsection{Simulation Method and Geometry}

The programing of simulation involved building a main application with different classes, namely, action initialization, primary generator, detector constructor, run and run action (https://indico.cern.ch/event/865808/page/19021-geant4-virtual-machine, assessed date: 2 November 2021). The action initialization class contained a class header (.hh files) and source (.cc files), and the class was used to instantiate and register to the Geant4 kernel. The primary generator class used a script to specify how a primary event is generated. The generator class defined the beam type (proton), beam direction (particle gun), beam energy, beam field size and distance between the beam source and gold nanoparticle. The detector constructor class defined the geometry environment of the MC simulation. The detector constructor source included the gold nanoparticle size and shape, as well as the distance between the gold nanoparticle and DNA. The material of the gold nanoparticle could be changed to gold (G4_Au) or water (G4_WATER) to investigate the effectiveness of adding a gold nanoparticle as a radiosensitizer in the medium. Moreover, detectors were set in this class. The Livermore low-energy physics models were used to simulate the interactions between protons and the gold nanoparticle, while the de-excitation cascade model was used for the secondary electrons produced by the gold nanoparticle [33]. In this study, the DNA base pair was defined as a dose detector to observe the total dose absorbed by the DNA. The cellular medium is assumed water equivalent. The run class managed the run procedure of the simulation. The dose absorptions were measured for each radiation history and passed to the run action class. There were 500 million histories in each simulation. The run action class was the user's action for each running procedure. This class was designed to obtain the deposited dose from the run action which was then 
added to the previous results. After finishing all simulations, the total dose was shown as the result.

The main function script of the simulation was used to create the G4RunManager instance to control the program's flow and manage the event loop. Through the creation of the run manager, the information to build and run the simulation was provided. This information included how the detector should be created, how all the particles and all the physics processes were to be simulated, how the primary particle in an event should be created, and any additional simulation requirements. In this study, the hadron therapy physics list was used for the simulation [34]. The physics list included many necessary processes for proton interactions with matter, such as the Bremsstrahlung effect, elastic scattering and inelastic scattering. The hadron therapy physics list was provided with the pre-packaged virtual machine Geant4 files.

The simulation geometry is shown in Figure 1.

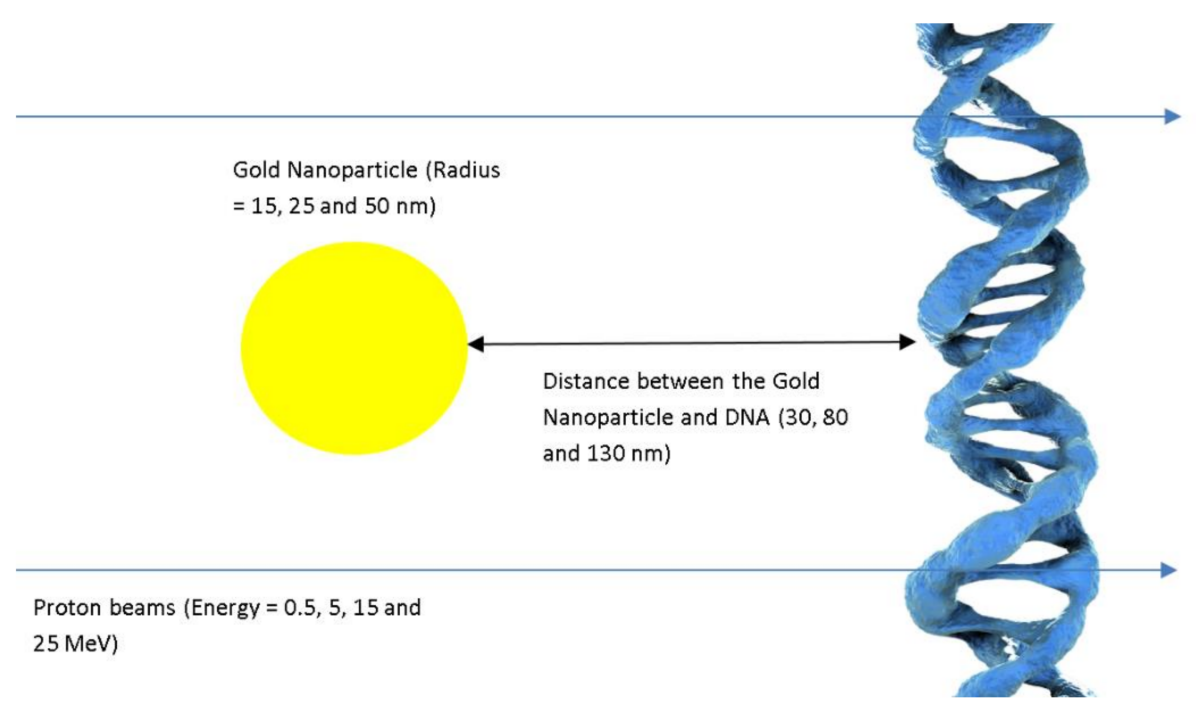

Figure 1. Schematic diagram showing the MC simulation geometry in Geant4-DNA (not to scale). The gold nanoparticle was located between the proton beam source and DNA molecule. Nanoparticle radii of 15,25 and $50 \mathrm{~nm}$ were used in the simulation. The distance between the nanoparticle and DNA was equal to 30,80 and $130 \mathrm{~nm}$, and the proton beam energy was equal to $0.5,5,15$ and $25 \mathrm{MeV}$.

In the simulation geometry, the DNA model as per Henthorn et al. was used [35] and defined with a single gold nanoparticle inside a spherical water phantom with a radius of $0.5 \mu \mathrm{m}$ [36]. The nanoparticle was positioned at the center of the phantom with the proton beam source on the left and the DNA on the right as shown in Figure 1. The proton source field (proton beam diameter) was a circular plane with a diameter of twice the diameter of the nanoparticle. It was positioned $200 \mathrm{~nm}$ away from the nanoparticle. The proton flux was irradiated as parallel beams onto the gold nanoparticle with different distances (30, 80 and $130 \mathrm{~nm}$ ) from the DNA molecule. Different nanoparticle radii of 15, 25 and $50 \mathrm{~nm}$ and different proton beam energies of $0.5,5,15$ and $25 \mathrm{MeV}$ were used. The gold nanoparticle coating was not applied in the simulation because our previous results showed that such coating around the gold nanoparticle does not affect the DNA dosimetry significantly [37].

\subsection{Dose Enhancement Ratio}

The DER in this study is defined as [26]:

$$
\text { Dose Enhancement Ratio }(\mathbf{D E R})=\frac{\text { Dose at DNA with Gold Nanoparticle }}{\text { Dose at DNA without Gold Nanoparticle }}
$$


The DER value reflects the enhancement of energy deposition at the DNA, resulting in a DNA damage, when a gold nanoparticle is present in the simulation. In Equation (1), the dose at DNA without a gold nanoparticle can be modeled in the simulation as replacing the material of gold (G4_Au) with water (G4_WATER) in the nanoparticle. In this case, both the nanoparticle and phantom were made up of water resulting in the DNA directly irradiated by the proton beam. It should be noted that a DER value larger than one means a dose enhancement when a gold nanoparticle is added to the phantom.

\section{Results}

The relationship of DER and proton beam energy $(0.5-25 \mathrm{MeV})$ is shown in Figure 2. Figure $2 \mathrm{a}-\mathrm{c}$ show the variations of DER vs. proton beam energy on the gold nanoparticle size (radius $=15,25$ and $50 \mathrm{~nm}$ ) and distance between the nanoparticle and DNA (30, 80 and $130 \mathrm{~nm}$ ). The uncertainty of the DER is less than $2 \%$ in the calculation.

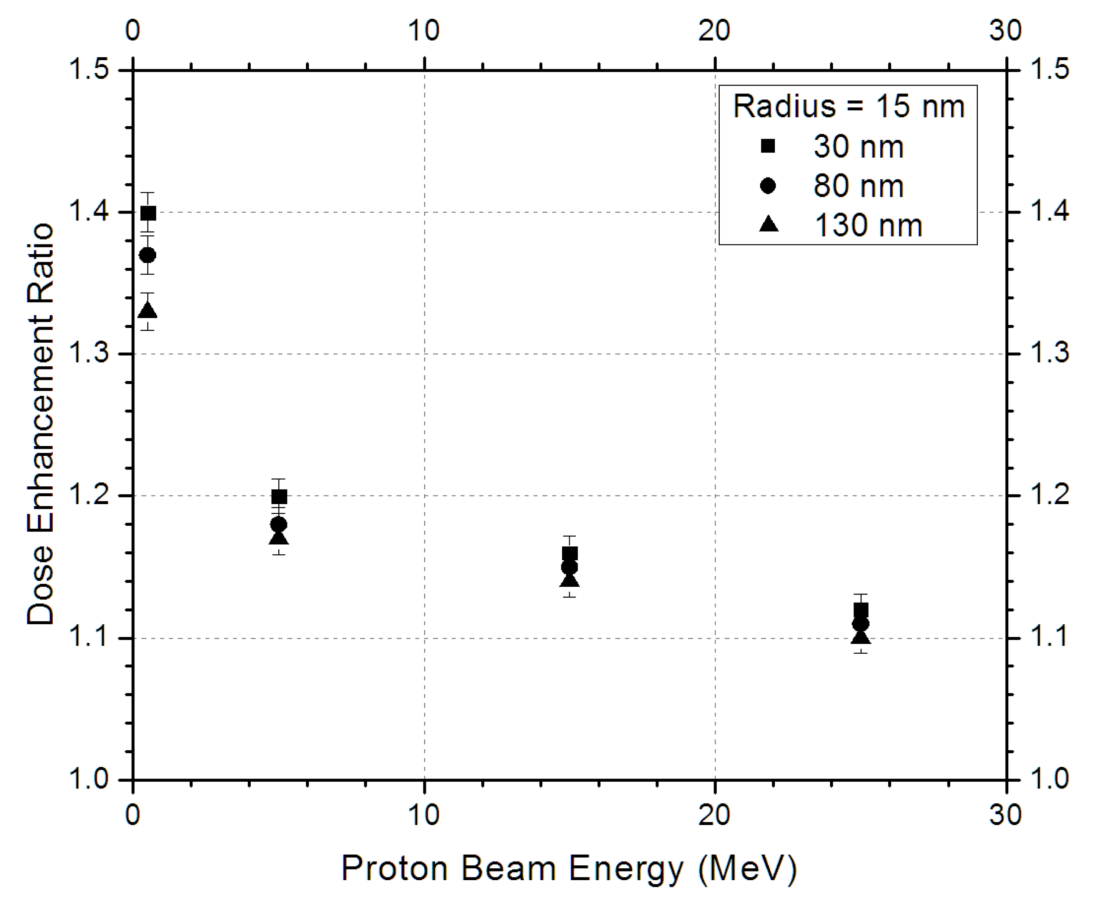

(a)

Figure 2. Cont. 


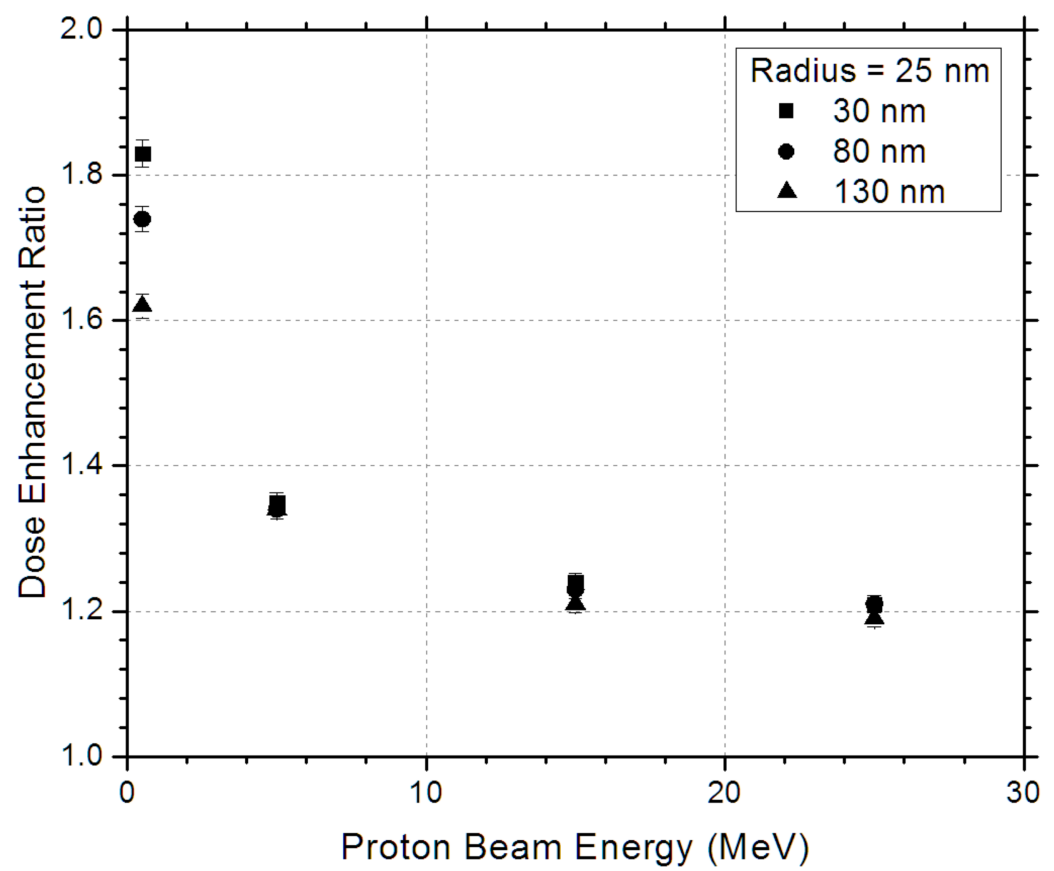

(b)

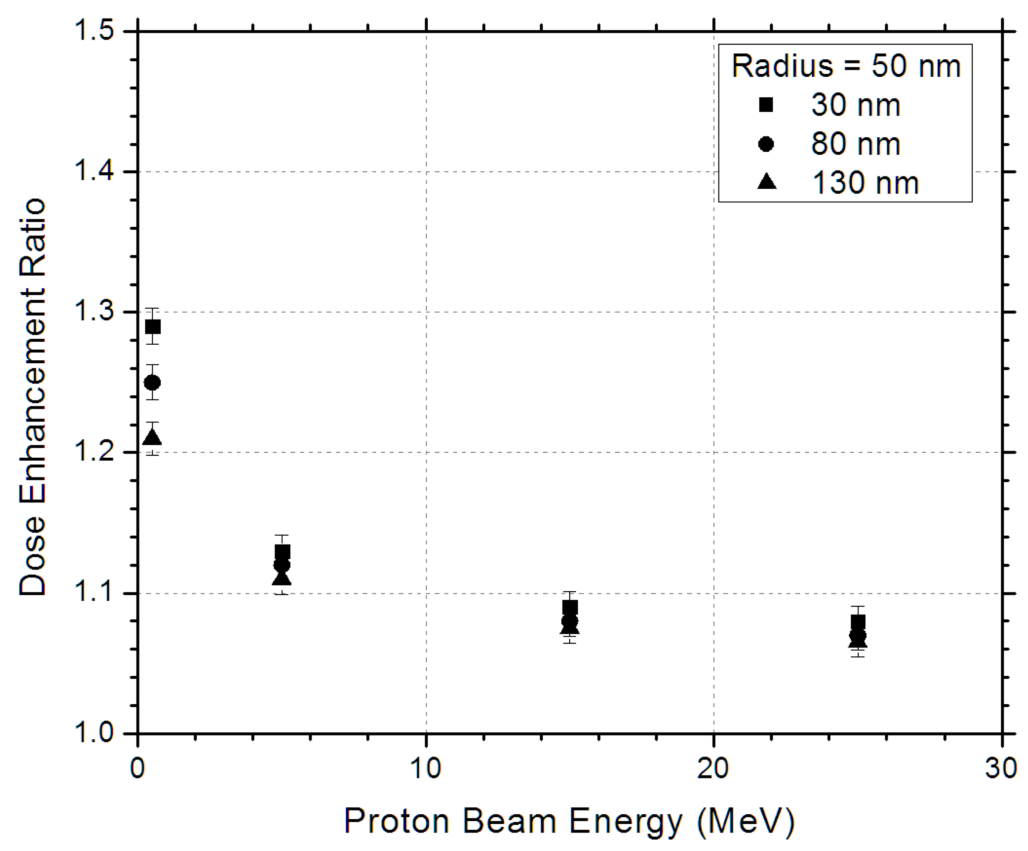

(c)

Figure 2. Dependence of dose enhancement ratio on proton beam energy (0.5-25 MeV). The distance between the gold nanoparticle and DNA was changed to 30,80 and $130 \mathrm{~nm}$, while the nanoparticle radius was changed to (a) 15, (b) 25 and (c) $50 \mathrm{~nm}$ in MC simulations. 


\section{Discussion}

\subsection{Dependence of DER on Gold Nanoparticle Size}

In Figure $2 \mathrm{a}-\mathrm{c}$, the maximum DER values are found at the minimum proton beam energy $(0.5 \mathrm{MeV})$ and minimum distance between the gold nanoparticle and DNA $(30 \mathrm{~nm})$. The maximum DER value is equal to 1.4 for the nanoparticle size of $15 \mathrm{~nm}$ (radius) as shown in Figure 2a. This value is smaller than that of the nanoparticle size of $25 \mathrm{~nm}$ radius (1.83 in Figure $2 b$ ) and larger than that of the nanoparticle size of $50 \mathrm{~nm}$ (1.29 in Figure 2c). It is seen that the DER value increases from a nanoparticle size of $15 \mathrm{~nm}$ to $25 \mathrm{~nm}$ and then decreases from $25 \mathrm{~nm}$ to $50 \mathrm{~nm}$. When the nanoparticle size increases from $15 \mathrm{~nm}$ to $25 \mathrm{~nm}$, the larger nanoparticle has a larger gold mass and particle volume for the interaction with the proton beam. This results in a larger yield of secondary electrons from the gold nanoparticle, and these electrons move to the DNA and cause dose enhancement. Therefore, the DER value increases. However, when the nanoparticle size further increases from 25 to $50 \mathrm{~nm}$, the self-absorption effect of the nanoparticle becomes significant. This larger nanoparticle starts to absorb more secondary electrons generated within the nanoparticle [38]. Moreover, the larger nanoparticle would cause more proton beam attenuation, leading to a decrease of dose enhancement at the DNA. This dependence of DER on gold nanoparticle size is also observed by Peukert et al. [39] using a different simulation geometry.

\subsection{Dependence of DER on Distance between the Gold Nanoparticle and DNA}

In the simulation, the distance between the gold nanoparticle and DNA varied in the range of 30 to $130 \mathrm{~nm}$ as shown in Figure 2. It is seen that the DER value decreases with an increase of distance between the nanoparticle and DNA. This is reasonable because the closer the gold nanoparticle to the DNA, the higher probability that the secondary electrons from the nanoparticle can reach the DNA and contribute energy deposition. This results in a larger DER value. Comparing Figure $2 \mathrm{a}-\mathrm{c}$, it is found that the variation of DER value on the distance between the nanoparticle and DNA is at maximum for the $0.5 \mathrm{MeV}$ proton beam. The DER range is equal to 1.33-1.4, 1.62-1.83, and 1.21-1.29, when the distance increases from 30-130 $\mathrm{nm}$ for the nanoparticle size (radius) equal to 15, 25 and $50 \mathrm{~nm}$, respectively. This change of DER value is not significant when compared to higher proton beam energy $(>0.5 \mathrm{MeV})$ with different nanoparticle sizes. It is seen that the effect of distance between the gold nanoparticle and DNA on the DER value is larger when lower proton beam energy is used to irradiate the nanoparticle. This is because the secondary electron range for the lower proton beam energy is shorter than the higher energy. This may cause some secondary electrons to be unable to reach the DNA when the nanoparticle is far away from the DNA molecule.

\subsection{Dependence of DER on Proton Beam Energy}

Considering the simulation geometry of the gold nanoparticle with size of $25 \mathrm{~nm}$ (radius) and a distance of $80 \mathrm{~nm}$ from the DNA (Figure 2a), it is found that the DER value decreases from 1.74 to 1.21 with an increase of proton beam energy. The higher energy deposition at the DNA with lower proton beam energy is due to the energy of secondary electrons. These low-energy secondary electrons from the gold nanoparticle, irradiated by the low-energy proton beam, cause more electron scatter between the nanoparticle and DNA molecule. This leads to more secondary electrons to reach the DNA and produce energy deposition. When a high-energy proton beam irradiates the gold nanoparticle, the secondary electrons generated have higher energy and may pass through the DNA without energy deposition. It should be noted that as the DNA is positioned in the downstream of the proton source behind the gold nanoparticle, and only the secondary electrons moving towards the DNA direction (i.e., from left to right in Figure 1) would cause a dose enhancement and therefore an increase of DER value. In addition, it can be seen from Figure 2 that the decrease of DER value from the low-energy proton beam (e.g., from 0.5 to $5 \mathrm{MeV}$ ) is more significant than the high-energy beam (e.g., 5 to $25 \mathrm{MeV}$ ). This is because the secondary electron scatter is particularly more significant at a low proton 
energy $(0.5 \mathrm{MeV})$. When the proton energy is in the range of 5 to $25 \mathrm{MeV}$, the decrease of DER value is only slight, as shown in Figure 2.

\section{Conclusions}

MC simulation was carried out to investigate the DNA dosimetry due to dose enhancement with a gold nanoparticle irradiated by a proton beam. DER was calculated based on the doses at the DNA with and without the presence of the gold nanoparticle. From the MC results, it is concluded that the variation of dose enhancement at the DNA depends on the gold nanoparticle size, distance between the nanoparticle and DNA, and proton beam energy. It is found that the dependence of DER value on the nanoparticle size is related to the gold mass/volume and to the self-absorption of the nanoparticle. Moreover, the DER value increases with a decrease of the distance between the nanoparticle and DNA, and a decrease of proton beam energy. Future work will include determining the dose distribution (2D/3D) within the DNA to estimate the DNA repair after the beam delivery, and studying the dependences of dose enhancement on the self-absorbed electrons, electron energy spectrum and nanoparticle cluster $[40,41]$.

Author Contributions: Conceptualization, J.C.L.C.; methodology, J.C.L.C. and N.H.H.; software, N.H.H.; validation, J.C.L.C. and N.H.H.; formal analysis, N.H.H.; investigation, J.C.L.C. and N.H.H.; resources, N.H.H.; data curation, N.H.H.; writing—original draft preparation, N.H.H.; writingreview and editing, J.C.L.C.; visualization, J.C.L.C. and N.H.H.; supervision, J.C.L.C.; project administration, J.C.L.C. All authors have read and agreed to the published version of the manuscript.

Funding: This research received no external funding.

Institutional Review Board Statement: Not applicable.

Informed Consent Statement: Not applicable.

Acknowledgments: The authors would like to thank Chun He and Kaden Kujanpaa from the University of Toronto, Canada, and Mehwish Jabeen from the Ryerson University, Canada, for their assistance in the MC simulation using the Geant4-DNA code.

Conflicts of Interest: The author declares no conflict of interest.

\section{References}

1. Public Health Agency of Canada. Cancer in Canada. 2018. Available online: https://www.canada.ca/content/dam/ phac-aspc/documents/services/publications/diseases-conditions/fact-sheet-cancer-canada/fact-sheet-cancer-canada.pdf (accessed on 28 September 2021).

2. Howlader, N.; Noone, A.M.; Krapcho, M.; Miller, D.; Brest, A.; Yu, M.; Ruhl, J.; Tatalovich, Z.; Mariotto, A.; Lewis, D.R.; et al. SEER Cancer Statistics Review, 1975-2018; National Cancer Institute: Bethesda, MD, USA, 2021. Available online: https:/ / seer.cancer.gov/csr/1975_2018/ (accessed on 28 September 2021).

3. Baskar, R.; Dai, J.; Wenlong, N.; Yeo, R.; Yeoh, K.W. Biological response of cancer cells to radiation treatment. Front. Mol. Biosci. 2014, 1, 24. [CrossRef]

4. Her, S.; Jaffray, D.A.; Allen, C. Gold nanoparticles for applications in cancer radiotherapy: Mechanisms and recent advancements. Adv. Drug Del. Rev. 2017, 109, 84-101. [CrossRef]

5. Moore, J.A.; Chow, J.C.L. Recent progress and applications of gold nanotechnology in medical biophysics using artificial intelligence and mathematical modeling. Nano Exp. 2021, 2, 022001. [CrossRef]

6. Siddique, S.; Chow, J.C.L. Application of nanomaterials in biomedical imaging and cancer therapy. Nanomaterials 2020, 10, 1700. [CrossRef]

7. Sung, W.; Ye, S.J.; McNamara, A.L.; McMahon, S.J.; Hainfeld, J.; Shin, J.; Smilowitz, H.M.; Paganetti, H.; Schuemann, J. Dependence of gold nanoparticle radiosensitization on cell geometry. Nanoscale 2017, 9, 5843-5853. [CrossRef]

8. Siddique, S.; Chow, J.C.L. Gold nanoparticles for drug delivery and cancer therapy. Appl. Sci. 2020, 10, 3824. [CrossRef]

9. Chithrani, D.B.; Jelveh, S.; Jalali, F.; Van Prooijen, M.; Allen, C.; Bristow, R.G.; Hill, R.P.; Jaffray, D.A. Gold nanoparticles as radiation sensitizers in cancer therapy. Radiat. Res. 2010, 173, 719-728. [CrossRef]

10. Chithrani, D.B.; Stewart, J.; Allen, C.; Jaffray, D.A. Intracellular uptake, transport, and processing of nanostructures in cancer cells. Nanomed. Nanotechnol. Biol. Med. 2009, 5, 118-127. [CrossRef]

11. Leung, M.K.; Chow, J.C.L.; Chithrani, B.D.; Lee, M.J.; Oms, B.; Jaffray, D.A. Irradiation of gold nanoparticles by x-rays: MC simulation of dose enhancements and the spatial properties of the secondary electrons production. Med. Phys. 2011, 38, 624-631. [CrossRef] 
12. Chow, J.C.L. Depth dose enhancement on flattening-filter-free photon beam: A MC Study in nanoparticle-enhanced radiotherapy. Appl. Sci. 2020, 10, 7052. [CrossRef]

13. Butterworth, K.T.; Nicol, J.R.; Ghita, M.; Rosa, S.; Chaudhary, P.; McGarry, C.K.; McCarthy, H.O.; Jimenez-Sanchez, G.; Bazzi, R.; Roux, S.; et al. Preclinical evaluation of gold-DTDTPA nanoparticles as theranostic agents in prostate cancer radiotherapy. Nanomedicine 2016, 11, 2035-2047. [CrossRef] [PubMed]

14. Hainfeld, J.F.; Smilowitz, H.M.; O'Connor, M.J.; Dilmanian, F.A.; Slatkin, D.N. Gold nanoparticle imaging and radiotherapy of brain tumors in mice. Nanomedicine 2013, 8, 1601-1609. [CrossRef]

15. Ali, M.R.; Wu, Y.; El-Sayed, M.A. Gold-nanoparticle-assisted plasmonic photothermal therapy advances toward clinical application. J. Phys. Chem. C 2019, 123, 15375-15393. [CrossRef]

16. Koonce, N.A.; Quick, C.M.; Hardee, M.E.; Jamshidi-Parsian, A.; Dent, J.A.; Paciotti, G.F.; Nedosekin, D.; Dings, R.P.; Griffin, R.J. Combination of gold nanoparticle-conjugated tumor necrosis factor- $\alpha$ and radiation therapy results in a synergistic antitumor response in murine carcinoma models. Int. J. Radiat. Oncol. Biol. Phys. 2015, 93, 588-596. [CrossRef] [PubMed]

17. Sung, W.; Schuemann, J. Energy optimization in gold nanoparticle enhanced radiation therapy. Phys. Med. Biol. $2018,63,135001$. [CrossRef] [PubMed]

18. Santivasi, W.L.; Xia, F. Ionizing radiation-induced DNA damage, response, and repair. Antioxidants Redox Signal. 2014, 21, 251-259. [CrossRef]

19. Chow, J.C.L. Photon and electron interactions with gold nanoparticles: A MC study on gold nanoparticle-enhanced radiotherapy. In Nanobiomaterials in Medical Imaging: Applications of Nanobiomaterials; Grumezescu, A.M., Ed.; Elsevier: Amsterdam, The Netherlands, 2016; pp. 45-70.

20. Mohan, R.; Grosshans, D. Proton therapy-present and future. Adv. Drug Del. Rev. 2017, 109, 26-44. [CrossRef] [PubMed]

21. Wälzlein, C.; Scifoni, E.; Krämer, M.; Durante, M. Simulations of dose enhancement for heavy atom nanoparticles irradiated by protons. Phys. Med. Biol. 2014, 59, 1441-1458. [CrossRef]

22. Martínez-Rovira, I.; Prezado, Y. Evaluation of the local dose enhancement in the combination of proton therapy and nanoparticles. Med. Phys. 2015, 42, 6703-6710. [CrossRef]

23. Lin, Y.; McMahon, S.J.; Scarpelli, M.; Paganetti, H.; Schuemann, J. Comparing gold nano-particle enhanced radiotherapy with protons, megavoltage photons and kilovoltage photons: A MC simulation. Phys. Med. Biol. 2014, 59, 7675-7689. [CrossRef]

24. Lin, Y.; McMahon, S.J.; Paganetti, H.; Schuemann, J. Biological modeling of gold nanoparticle enhanced radiotherapy for proton therapy. Phys. Med. Biol. 2015, 60, 4149-4168. [CrossRef]

25. Lin, Y.; Paganetti, H.; McMahon, S.J.; Schuemann, J. Gold nanoparticle induced vasculature damage in radiotherapy: Comparing protons, megavoltage photons, and kilovoltage photons. Med. Phys. 2015, 42, 5890-5902. [CrossRef]

26. Chow, J.C.L. Dose enhancement effect in radiotherapy: Adding gold nanoparticles to tumor in cancer treatment. In Nanostructures for Cancer Therapy; Elsevier: Amsterdam, The Netherlands, 2017; pp. 383-403.

27. Rogers, D.W.O. Fifty years of MC simulations for medical physics. Phys. Med. Biol. 2006, 51, R287. [CrossRef] [PubMed]

28. Chow, J.C.L. Recent progress in MC simulation on gold nanoparticle radiosensitization. AIMS Biophys. 2018, 5, 231-244. [CrossRef]

29. Incerti, S.; Baldacchino, G.; Bernal, M.; Capra, R.; Champion, C.; Francis, Z.; Gueye, P.; Mantero, A.; Mascialino, B.; Moretto, P.; et al. The geant4-dna project. Int. J. Model. Simulat. Sci. Comp. 2010, 1, 157-178. [CrossRef]

30. Hoffman, W.; Martin, K. The CMake Build Manager. Dr. Dobb's J. Softw. Tools Prof. Program. 2003, $28,40-43$.

31. Indico. Getting Started with Geant4. 2021. Available online: https://indico.cern.ch/event/865808/page/19021-geant4-virtualmachine (accessed on 28 September 2021).

32. Bugnion, E.; Devine, S.; Rosenblum, M.; Sugerman, J.; Wang, E.Y. Bringing virtualization to the $\mathrm{x} 86$ architecture with the original vmware workstation. ACM Trans. Comput. Syst. (TOCS) 2012, 30, 1-51. [CrossRef]

33. Incerti, S.; Suerfu, B.; Xu, J.; Ivantchenko, V.; Mantero, A.; Brown, J.M.; Bernal, M.A.; Francis, Z.; Karamitros, M.; Tran, H.N. Simulation of Auger electron emission from nanometer-size gold targets using the Geant4 MC simulation toolkit. Nucl. Instrum. Methods Phys. Res. Sect. B Beam Interact. Mater. Atoms. 2016, 372, 91-101. [CrossRef]

34. Cirrone, G.A.; Cuttone, G.; Mazzaglia, E.S.; Romano, F.; Sardina, D.; Agodi, C.; Attili, A.; Blancato, A.A.; De Napoli, M.; Di Rosa, F.; et al. Hadrontherapy: A Geant4-based tool for proton/ion-therapy studies. Prog. Nucl. Sci. Technol. 2011, 2, 207-212. [CrossRef]

35. Henthorn, N.T.; Warmenhoven, J.W.; Sotiropoulos, M.; Mackay, R.I.; Kirkby, K.J.; Merchant, M.J. Nanodosimetric simulation of direct ion-induced DNA damage using different chromatin geometry models. Radiat. Res. 2017, 188, 690-703. [CrossRef]

36. Jabeen, M.; Chow, J.C.L. Gold nanoparticle DNA damage by photon beam in a magnetic field: A MC study. Nanomaterials 2021, 11, 1751. [CrossRef] [PubMed]

37. Chun, H.; Chow, J.C.L. Gold nanoparticle DNA damage in radiotherapy: A MC study. AIMS Bioeng. $2016,3,352-361$.

38. Chow, J.C.L.; Leung, M.K.; Jaffray, D.A. MC simulation on a gold nanoparticle irradiated by electron beams. Phys. Med. Biol. 2012, 57, 3323. [CrossRef]

39. Peukert, D.; Kempson, I.; Douglass, M.; Bezak, E. Gold nanoparticle enhanced proton therapy: A MC simulation of the effects of proton energy, nanoparticle size, coating material, and coating thickness on dose and radiolysis yield. Med. Phys. 2020, 47, 651-661. [CrossRef] [PubMed] 
40. Ahn, S.H.; Chung, K.; Shin, J.W.; Cheon, W.; Han, Y.; Park, H.C.; Choi, D.H. Study on dependence of dose enhancement on cluster morphology of gold nanoparticles in radiation therapy using a body-centred cubic model. Phys. Med. Biol. 2017, 62, 7729. [CrossRef] [PubMed]

41. Kirkby, C.; Koger, B.; Suchowerska, N.; McKenzie, D.R. Dosimetric consequences of gold nanoparticle clustering during photon irradiation. Med. Phys. 2017, 44, 6560-6569. [CrossRef] 